\title{
Extraction of Neutrino Flux from the Inclusive Muon Cross Section
}

Tomoya Murata*

Osaka University

E-mail: muratadkern.phys.sci.osaka-u.ac.jp

Toru Sato

Osaka University

E-mail: tsatodphys.sci.osaka-u.ac.jp

We have studied a method to extract neutrino flux from the data of neutrino-nucleus reaction by using maxmum entropy method. We demonstrate a promising example to extract neutrino flux from the inclusive cross section of muon production without selecting a particular reaction process such as quasi-elastic nucleon knockout.

16th International Workshop on Neutrino Factories and Future Neutrino Beam Facilities 25 -30 August, 2014

University of Glasgow, United Kingdom

\footnotetext{
* Speaker.
} 


\section{Introduction}

A precise description of the neutrino-nucleus interaction is crucial for the study the neutrino

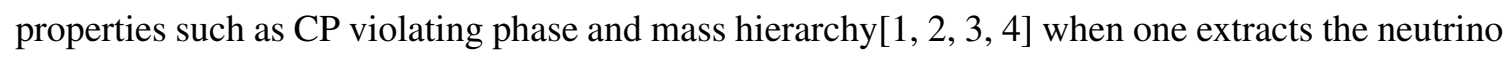
flux from the neutrino data. The quasi-elastic $(\mathrm{QE})$ reaction is the key mechanism to reconstruct the neutrino flux from the data of neutrino-nucleus reaction. Neutrino energy $\left(E_{v}\right)$ can be determined from the muon scattering angle $(\theta)$ and energy $\left(E_{\mu}\right)$ by using the kinematics of the reaction of free nucleon at rest.

$$
E_{v}=\frac{E_{\mu} M_{N}-m_{\mu}^{2} / 2}{M_{N}-E_{\mu}+p_{\mu} \cos \theta}
$$

Since the reaction takes place inside nuclear many body system, various initial and final state interactions can spoil the useful relation of Eq. (ㅍ. $)$. The observed quasi-elastic like events are mixture of the various reaction mechanism, which contribute the reaction amplitudes and, in principle, interference among various mechanism exists. The central issue of the current theoretical research of neutrino-nucleus reaction is to improve the accuracy of the estimation of various nuclear effects[[1, 6] by testing the model from the neutrino data.

Because of the rather wide energy band of the neutrino flux in the current long baseline experiments, the observed cross section of neutrino reaction $(\langle\sigma\rangle)$ is a result of neutrino flux $\left(\Phi_{v}\right)$ average of the cross section $(\sigma)$.

$$
<\sigma>=\int d E_{v} \sigma\left(E_{v}\right) \Phi_{v}\left(E_{v}\right)
$$

One can regard the extraction of neutrino flux $\Phi_{v}$ from the data $\langle\sigma\rangle$ as an inverse problem to obtain $\Phi_{v}$ from $\langle\sigma\rangle$ provided the cross section $\sigma$ is well under control. In this report we will examine a method to solve the inverse problem, where the neutrino flux can be extracted without using the formula (ㅍ. $)$ nor the separation of 'true' QE events. For this purpose, we use the maximum entropy method (MEM) which are widely used in the field of condensed matter physics and Lattice QCD[D, [8, Q Q].

We briefly outline the application of MEM to extract neutrino flux from data in section 2. To examine the usefulness of the approach, we generate pseudo data of neutrino reaction. The pseudo data are generated by using a model of neutrino reaction and neutrino flux, which are explained in section 3. Here, as an example of the pseudo data, we use double differential cross section of muon for the inclusive neutrino nucleus reaction. Results on how well the neutrino flux can be extracted from the pseudo 'data' without assuming QE mechanism is shown in section 4.

\section{Maximum Entropy Method}

In this section we briefly explain the maximum entropy method. The most plausible neutrino flux $\left(\Phi_{v}^{M E M}\right)$ from the experimental data based on the Bayes' theorem is given by the functional integral of the neutrino flux $\Phi$

$$
\Phi_{v}^{M E M}=\int[d \Phi] \Phi P[\Phi \mid \bar{D}, I]
$$


where $P[\Phi \mid \bar{D}, I]$ is a conditional probability of neutrino flux $\Phi$ for given data of neutrino reaction $\bar{D}=\left\{\langle\sigma\rangle_{\text {exp }}\right\}$ and the prior information on the neutrino flux $I . I$ is called as default model of neutrino flux. Introducing auxiliary variable $\alpha$, we can write the above formula as

$$
\Phi_{v}^{M E M}=\int d \alpha \Phi_{v}^{\alpha} P[\alpha \mid \bar{D}, I]
$$

where $\Phi_{v}^{\alpha}$ is neutrino flux for given $\alpha$, data $\bar{D}$ and prior information $I$ given as,

$$
\Phi_{v}^{\alpha}=\int[d \Phi] \Phi P[\Phi \mid \alpha, \bar{D}, I] \propto \int[d \Phi] \Phi P[\bar{D} \mid \Phi, \alpha, I] P[\Phi \mid \alpha, I] .
$$

Here $P[\bar{D} \mid \Phi, \alpha, I]$ and $P[\Phi \mid \alpha, I]$ are called as likefood function and prior probability, respectively. The likefood function is written as

$$
P[\bar{D} \mid \Phi, I]=\frac{1}{Z_{\chi}} \exp \left(-\frac{1}{2} \chi^{2}\right)
$$

with

$$
\begin{aligned}
& \chi^{2}=\sum_{l=1} \frac{\left(\bar{D}_{l}-D_{l}\right)^{2}}{\sigma_{l}^{2}} \\
& Z_{\chi}=\prod_{l=1} \sqrt{2 \pi \sigma_{l}^{2}} .
\end{aligned}
$$

Here $\bar{D}_{l}$ and $D_{l}$ are cross sections $\left\langle\sigma>_{\text {exp }}\right.$ and $\langle\sigma\rangle$.

The prior probability can be expressed as

$$
P[\Phi \mid \alpha, I]=\frac{1}{Z_{S}} \exp (\alpha S),
$$

where $\mathrm{S}$ is the Shannon-Jaynes entropy,

$$
S=\sum_{i}\left(\Phi_{i}-m_{i}-\Phi_{i} \ln \frac{\Phi_{i}}{m_{i}}\right) .
$$

$m_{i}$ is default model of neutrino flux at neutrino energy $E_{i}$. Combining the likefood function and the prior probability, neutrino flux $\Phi_{v}^{\alpha}$ is the maximum probability of $P[\Phi \mid \alpha, \bar{D}, I] \propto e^{Q(\Phi)}$ with $Q(\Phi)=\alpha S-\frac{1}{2} \chi^{2}$

Finally the $\alpha$ dependent flux is integrated with the probability $P[\alpha \mid \bar{D}, I]$, which has a sharp peak as a function of $\alpha$ written as

$$
P[\alpha \mid \bar{D}, I]=\int[d \Phi] P[\Phi \alpha \mid \bar{D}, I] \propto P[\alpha \mid I] \int[d \Phi] \frac{1}{Z_{S} Z_{\chi}} e^{Q(\Phi)}
$$

Assuming constant $P[\alpha \mid I]$, we obtain the neutrino flux $\Phi_{v}^{M E M}$ by integrating $\alpha$ around the shark peak of $P[\alpha \mid \bar{D}, I]$ in Eq. $\overline{2.2}$.

One can also estimate the errors of the extracted neutrino flux $\Phi_{v}^{M E M}$ within MEM. For given $\alpha$, the average flux $<\Phi_{\alpha}>_{S}$ in energy region $S$ of neutrino is defined as

$$
\left\langle\Phi_{v}^{\alpha}\right\rangle_{S}=\frac{\int_{S} d E_{v} \Phi_{v}^{\alpha}\left(E_{v}\right)}{\int_{S} d E_{v}}
$$


The covariance of $\left\langle\Phi_{v}^{\alpha}\right\rangle_{S}$ can be written as

$$
\begin{aligned}
\left\langle\left(\delta \Phi_{v}^{\alpha}\right)^{2}\right\rangle_{S} & =\int_{S^{2}} d E_{v} d E_{v}^{\prime} \delta \Phi\left(E_{v}\right) \Phi\left(E_{v}^{\prime}\right) P[\Phi \mid \bar{D}, \alpha, I] / \int_{S^{2}} d E_{v} d E_{v}^{\prime} \\
& \simeq-\int_{S^{2}} d E_{v} d E_{v}^{\prime}\left(\frac{\delta^{2} Q}{\delta \Phi\left(E_{v}\right) \delta \Phi\left(E_{v}^{\prime}\right)}\right)_{\Phi=\Phi_{\alpha}}^{-1} / \int_{S^{2}} d E_{v} d E_{v}^{\prime} .
\end{aligned}
$$

Then the error of the extracted flux is obtained by integrating over $\alpha$ as

$$
\left\langle\left(\delta \Phi_{v}^{M E M}\right)^{2}\right\rangle_{S}=\int d \alpha\left\langle\left(\delta \Phi_{v}^{\alpha}\right)^{2}\right\rangle_{S} P[\alpha \mid \bar{D}, I]
$$

\section{Model of inclusive neutrino-nucleus cross section and neutrino flux}

To examine the method outlined in the previous section, we construct pseudo data of neutrinonucleus reaction by using a model of neutrino-nucleus reaction and neutrino flux. The neutrino flux is obtained by applying the formula of two-flavor oscillation as

$$
\Phi\left(E_{v}\right)=\left(1-\sin ^{2} 2 \theta_{23} \sin ^{2}\left(\frac{\Delta m_{23} L}{E_{v}}\right)\right) \Phi_{v}^{0}\left(E_{v}\right)
$$

Here we used $\Delta m_{23}^{2}=2.5 \times 10^{-3} \mathrm{eV}^{2}, \sin ^{2} 2 \theta_{23}=1.0$ and $L=295 \mathrm{~km}$. The initial flux $\Phi_{v}^{0}\left(E_{v}\right)$ is taken from near detector neutrino flux of T2K experiment Ref. [ए]]. The solid(black) curve in Fig.

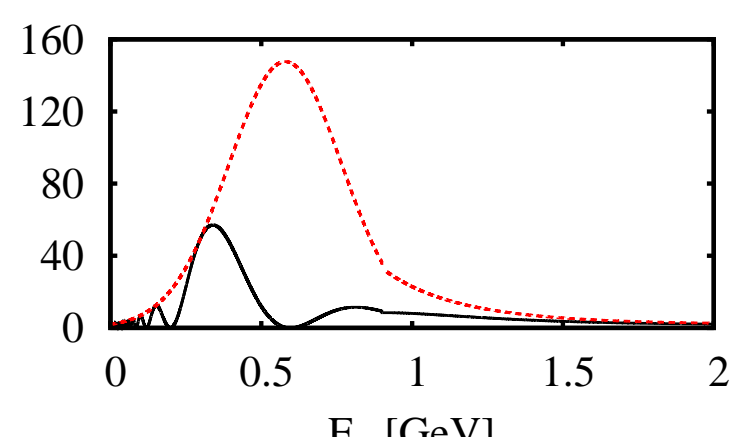

Figure 1: Neutrino flux. The solid(black') and the dashed(red) lines show the neutrino flux $\Phi_{v}$ and $\Phi_{v}^{0}$.

1 is the neutrino flux we use to calculate the pseudo data, while the original flux $\Phi_{v}^{0}$ is shown in dashed(red) curve for comparison.

As a observable to test the method of flux extraction, we use double differential cross section of muon of neutrino induced inclusive reaction on ${ }^{12} C, v_{\mu}+{ }^{12} C \rightarrow \mu^{-}+X$

$$
\frac{d^{2} \sigma}{d E_{\mu} d \Omega_{\mu}}
$$

In the energy region of neutrino flux in Fig. 1, the main reaction mechanism is quasi elastic scattering and pion production through the $\Delta(1232)$ excitation. The quasi elastic nucleon knockout 
process is calculated using modified Fermi-gass model which incorporates the spectral function [122, [13]. For the delta excitation region we use the neutrino pion production amplitudes calculated in a formalism in which the resonance contributions and the background amplitudes are treated on the same footing[ㄴ]]. Detail on the reaction model is described in Ref. [प]]. The model of nuclear reaction is rather simple without taking into account the final state interaction, however the reliability of our formalism is tested against the electron nucleus scattering cross sections in the same theoretical framework; the calculated cross sections agree reasonably well with the existing data. Therefore, we believe it is good enough for the purpose of testing the method of neutrino flux extraction.

We now are able to calculate the flux averaged double differential cross section $d^{2} \sigma / d E_{\mu} d \Omega_{\mu}$ from the model of neutrino-nucleus reaction and neutrino flux $\Phi_{v}$ in Eq. (3.1).

$$
\left\langle\frac{d^{2} \sigma}{d E_{\mu} d \Omega_{\mu}}\right\rangle=\int d E_{v} \frac{d^{2} \sigma}{d E_{\mu} d \Omega_{\mu}} \Phi\left(E_{v}\right)
$$

The pseudo data of double differential cross section is obtained as following. We assume muon energy resolution is $100 \mathrm{MeV}$ and evaluate the average of cross section as,

$$
\left\langle\overline{\frac{d^{2} \sigma}{d E_{\mu} d \Omega_{\mu}}}\right\rangle=\int_{E_{1}}^{E_{2}} d E_{\mu} \frac{1}{E_{2}-E_{1}}\left\langle\frac{d^{2} \sigma}{d E_{\mu} d \Omega_{\mu}}\right\rangle
$$

We assume Gaussian distribution of the data with the mean given by Eq. (3.4) and the standard deviation by the $10 \%$ of the value of flux averaged double differential cross section at $E_{\mu}=1 \mathrm{GeV}$. Fig. 2 shows the obtained pseudo data of the muon energy distribution at the muon scattering angle $\theta_{\mu}=10^{\circ}$.

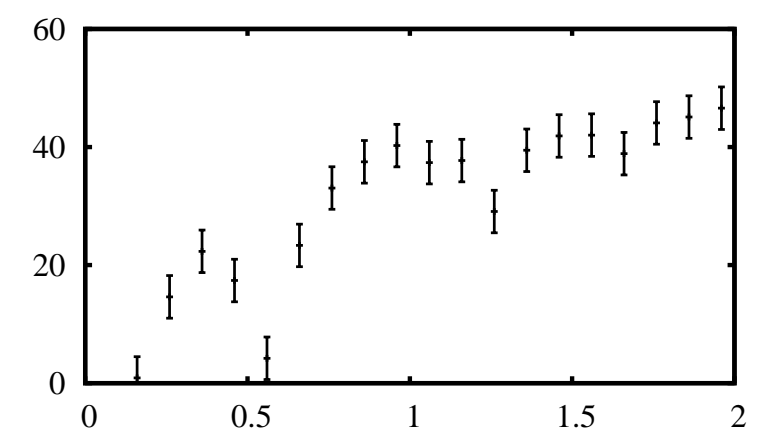

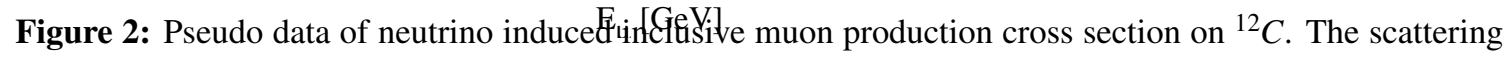
angle of the muon is $\theta_{\mu}=10^{\circ}$.

\section{Results and discussion}

We extract neutrino flux from the 19 points of pseudo data of the muon energy distribution shown in Fig. 2. They give $\bar{D}_{l}$ and $\sigma_{l}$ of Eq. (2.5). $D_{l}$ is calculated from the theoretical cross section of Eq. (3.2). 
At first, we study the effects of the default model $I$ on the extracted neutrino flux. We have examined two default models. The one is constant flux and the other is initial flux $\Phi_{v}^{0}$ shown in the dashed(red) and solid(black) curve in the left panel of Fig. 3. The extracted flux using each default
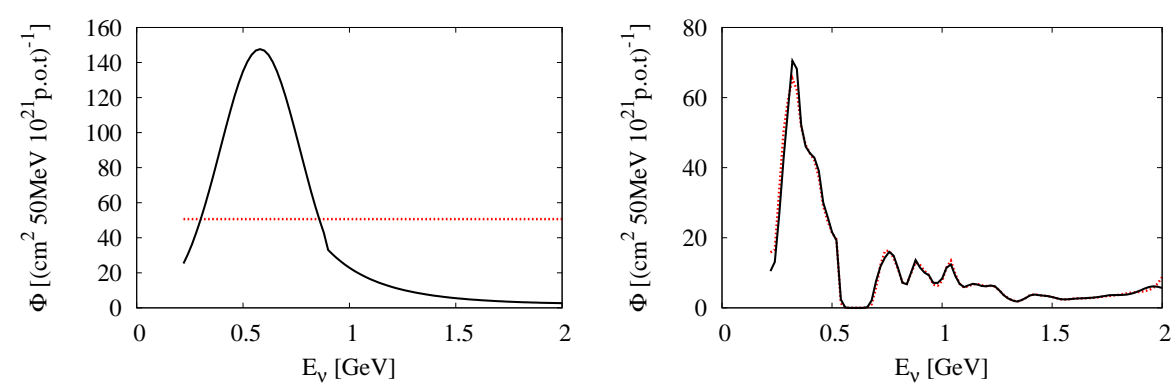

Figure 3: Default model dependence of extracted neutrino flux. The solid(black) and dashed(red) curves in the left panel show $\Phi_{v}^{0}$ and constant flux as default model. The extracted flux $\Phi_{v}^{M E M}$ corresponding to each default model is shown in the right panel.

model is shown in the right panel of Fig. 3. One can see that, in this case, the extracted flux is very stable against the choice of the default model.

We now compare the extracted flux $\Phi_{v}^{M E M}$ (solid curve) with the expected 'exact' neutrino flux $\Phi_{v}$ (dashed curve) in Fig. 4. Extracted flux agrees well with the 'exact' flux over the wide muon energy region. The extraction of the flux is only valid on the energy above the reaction threshold and the flux extraction did not work well near the threshold energy. We noticed that the extracted flux has structures in the $0.7<E_{v}<1 \mathrm{GeV}$ region. One can judge the structure is real

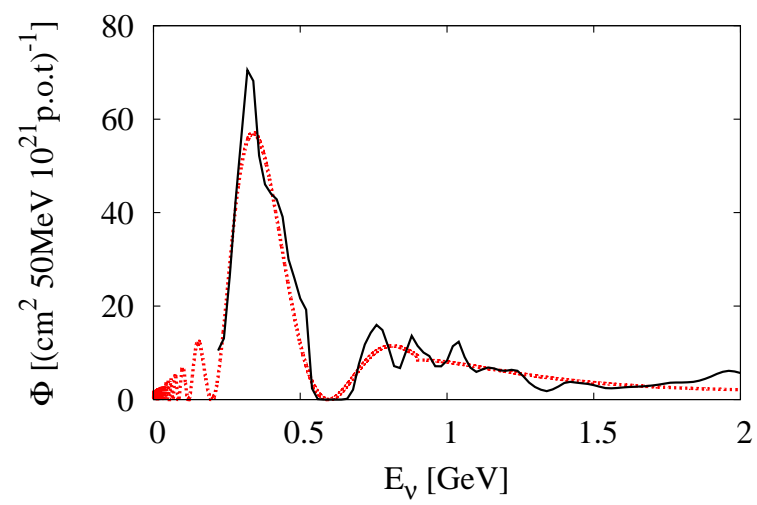

Figure 4: Comparison of extracted flux $\Phi_{v}^{M E M}$ (solid curve) and 'true' flux $\Phi_{v}$ (dashed curve).

or not by estimating the error of extracted flux. In Table 1, the neutrino flux averaged in the peak region $\left(0.28<E_{v}<0.42 \mathrm{GeV}\right)$, dip region $\left(0.52<E_{v}<0.70 \mathrm{GeV}\right)$ and higher energy regions $\left(0.72<E_{v}<0.82 \mathrm{GeV}\right),\left(0.86<E_{v}<0.94 \mathrm{GeV}\right)$ and $\left(1.00<E_{v}<1.08 \mathrm{GeV}\right)$ are given. The second raw shows the calculated 'exact' value of the average flux and the third raw shows the flux obtained from MEM together with the error calculated from Eq. (2.11). The central values of the 
$\Phi_{v}^{M E M}$ agree well with the 'exact' ones within the errors in all the energy regions. The size of the oscillation of $\Phi_{v}^{M E M}$ seen in $0.7<E_{v}<1 \mathrm{GeV}$ is smaller than the estimated errors in that region. Therefore one can conclude that we sould not take the oscillation of the obtained flux seriously from the error estimation even without knowing the 'exact' flux.

\begin{tabular}{cccccc}
\hline$E_{v}(\mathrm{GeV})$ & $0.28-0.42$ & $0.52-0.70$ & $0.72-0.82$ & $0.86-0.94$ & $1.00-1.08$ \\
Exact & 53.5 & 3.9 & 10.5 & 10.9 & 8.7 \\
MEM & $56.5 \pm 6.5$ & $5.1 \pm 1.7$ & $14.0 \pm 3.7$ & $11.6 \pm 3.7$ & $10.7 \pm 1.9$ \\
\hline
\end{tabular}

Table 1: Energy averaged neutrino flux.

In summary, we have examined MEM to extract the neutrino flux from the inclusive muon production cross section. The results show the proposed method can be useful alternative tool to extract the neutrino flux provided accurate theoretical cross section is known. Further work to find the optimal observables for the efficient extraction of flux with the refined model of neutrinonucleus reaction should be done in near future.

The authors would like thank to Drs. M. Kitazawa and S. Nakamura for illuminating discussions. This work was supported by JSPS KAKENHI Grant Nos. 24540273 and 25105010.

\section{References}

[1] K. Abe et al. (Hyper-Kamiokande working group), arXiv 1109.3262[hep-ex] (2011).

[2] C. Adams et al. (LBNE collaboration) arXiv:1307.7335[hep-ex] (2013).

[3] S. K. Agarwalla et al. (LAGUNA-LBNO collaboration), JHEP05, 094 (2014).

[4] S. Bhadra et al. (The nuPRISM Collaboration) arXiv:1412.3086 [physics.ins-det] (2014).

[5] L. Alvarez-Ruso, Y. Hayato and J. Nieves, New Jour. Phys. 16, 075015 (2014).

[6] G. T. Garvey, D. A. Harris, H. A. Tanaka, R. Tayloe and G. P. Zeller, arXiv:1412.4294[hep-ex] (2014).

[7] R. N. Silver, D. S. Sivia and J. E. Gubernatis, Phys. Rev. B41 2380 (1990).

[8] J. E. Gubernatis, M. Jarrell, R. N. Silver and D. S. Sivia, Phys. Rev. B44, 6011 (1991).

[9] M. Asakawa, T. Hatsuda and Y. Nakahara, Prog. Part. Nucl. Phys. 46459 (2001).

[10] K. Abe et al. (T2K Collaboration) Phys. Rev. D87 012001 (2013).

[11] B. Szczerbinska, T. Sato, K. Kubodera and T. -S. H. Lee, Phys. Lett. B649, 132 (2007).

[12] O. Benhar, A. Fabrocini, S. Fantoni and I. Sick, Nucl. Phys. A579 493 (1994).

[13] O.Benhar, N. Farina, H. Nakamura, M. Sakuda and R. Seki, Phys. Rev. D72 053005 (2005).

[14] T.Sato, D. Uno and T. -S. H. Lee, Phys. Rev. C67 065201 (2003). 